\title{
Development of Amplitude-Integrated Electroencephalography and Interburst Interval in the Rat
}

\author{
ALEXANDER M. TUCKER, KRISTIAN AQUILINA, ELA CHAKKARAPANI, CATHERINE E. HOBBS, \\ AND MARIANNE THORESEN
}

Department of Child Health, University of Bristol, Bristol, BS2 8EG, United Kingdom

\begin{abstract}
Continuous monitoring of electrocortical brain activity with amplitude-integrated electroencephalography (aEEG) is important in neonatology. aEEG is affected by, for example, maturity, encephalopathy, and drugs. Neonatal research uses rat pups of different ages. Postnatal day (P) 7 rats are suggested to be equivalent neurodevelopmentally to near-term infants. We hypothesized that electroencephalography (EEG) and aEEG in P1-P21 rats follow the same developmental pattern with respect to background activity and the longest interburst interval (IBI) as that seen in infants from 23-wk gestational age (GA) to post-term. We examined aEEG and EEG on 49 , unsedated rat pups with two clinical monitors. aEEG traces were analyzed for lower and upper margin amplitude, bandwidth and the five longest IBI in each trace were measured from the raw EEG. The median longest IBI decreased linearly with age by $5.24 \mathrm{~s} / \mathrm{d}$ on average. The lower border of the aEEG trace was $<5 \mu \mathrm{V}$ until P7 and rose exponentially reaching $10 \mu \mathrm{V}$ by $\mathrm{P} 12$. This correlated strongly with the decrease in IBI; both reflect increased continuity of brain activity with postnatal age. Based on aEEG trace analysis, the rat aEEG pattern at P1 corresponds to human aEEG at 23-wk gestation; P7 corresponds to 30-32 wk and P10 to 40-42 wk. (Pediatr Res 65: 62-66, 2009)
\end{abstract}

A mplitude-integrated electroencephalography (aEEG) is increasingly used in neonatology for continuous monitoring of cerebral electrical activity (1) in relation to brain injury, seizures, and assessment of brain maturation. aEEG has gained widespread popularity as an adjunct to conventional EEG because of its simplicity of application and interpretation and feasibility for long-term recording alongside clinical care. Although multi-channel, raw electroencephalography (EEG) is still the "gold standard" in assessing the brain's functional state, its usefulness in the newborn is limited for several practical reasons $(2,3)$. The assessment of background activity, presence of sleep wake cycling (SWC), and seizure identification, of particular importance in neonatology, require a longer recording period than the standard 30-min EEG. As global brain activity is the focus of interest in the newborn, one or two EEG channels are generally sufficient; thus reducing the number of electrodes attached to a small head.

Received July 9, 2008; accepted July 28, 2008.

Correspondence: Marianne Thoresen, M.D., Ph.D., St. Michael's Hospital, Child Health, Level D, Southwell Street, Bristol, BS2 8EG, United Kingdom; e-mail: Marianne.Thoresen@bristol.ac.uk

This work was supported by SPARKS UK and Olympic Medical, Seattle, WA, a division of Natus Medical Inc, and BrainZ Ltd., Auckland, New Zealand by lending the aEEG monitors.
Translational research, the use of animals to model human disease, is essential in developing effective treatments for human disorders. The newborn rat has proven invaluable in increasing our understanding of mechanisms of brain injury and repair processes (4-8). The Vanucci model, in which 7-d-old rats undergo unilateral ligation of the common carotid artery and exposure to $8 \%$ oxygen, is the most widely used animal model in the study of neonatal hypoxic-ischemic encephalopathy (HIE) (8); however, many models from postnatal day (P) 1 until P14 have been developed (5,8-10). The usefulness of animal models is contingent on their validity. Previous research has correlated the maturation of the rat to the human brain using various criteria including white matter and oligodendrocyte development, myelination, enzyme activity, synapse formation, structural changes in the brain, and EEG (11-13). However, the contribution of aEEG in correlating rat to human brain maturity has not been evaluated. In this study, we examined aEEG and the longest interburst interval (IBI) in EEG, in the newborn rat from P1 (day of birth) to P21 (equivalent in EEG maturation to a human of at least $2 \mathrm{y}$ of age) and compared it with published aEEG and EEG data from human neonates at different gestational ages (GAs).

\section{METHODS}

All procedures were conducted under Home Office license in accordance with UK regulations. Pregnant Wistar rats were bred at the University of Bristol animal facilities. Dams with litters were exposed to a 12:12 light:dark cycle with food and water available ad libitum.

Animal preparation. A temperature probe was inserted $5 \mathrm{~mm}$ rectally (calibration accuracy $<0.1^{\circ} \mathrm{C}$, Physitemp Instruments, Clifton, NJ), allowing core temperature to be monitored continuously. All recordings were made on a heating/cooling mattress (Tecotherm, Halle, Germany) which allowed core temperature to be maintained at $37.0 \pm 0.2^{\circ} \mathrm{C}$, considered normothermia for rat pups (14). Pups of this size are poikilothermic if not with the dam and have shown to not become hypoglycemic after separation from the dam for up to $12 \mathrm{~h}(8,15)$.

Recordings. Continuous, single-channel (CFM 6000, Olympic Medical, Seattle, WA = machine A) or two-channel (BrainZ Ltd., Auckland, New Zealand $=$ machine $\mathrm{B}$ ) $\mathrm{aEEG}$ and EEG was recorded on 49 rat pups, yielding 110 traces from P1 to P21 (56 traces from A and 54 from B). Five rats underwent one recording, 27 underwent two recordings, and 17 underwent three recordings using machines randomly assigned on each day throughout their survival period. Five millimeter subdermal stainless steel needle electrodes (Viasys Healthcare, Madison, WI) were positioned under brief halothane anesthesia (3.5\% induction). After anesthesia [median duration 5 min (range 4-7)],

Abbreviations: aEEG, amplitude-integrated electroencephalography; EEG, electroencephalography; GA, gestational age; HIE, hypoxic-ischemic encephalopathy; IBI, interburst interval; P, postnatal day; SWC, sleep wake cycling 


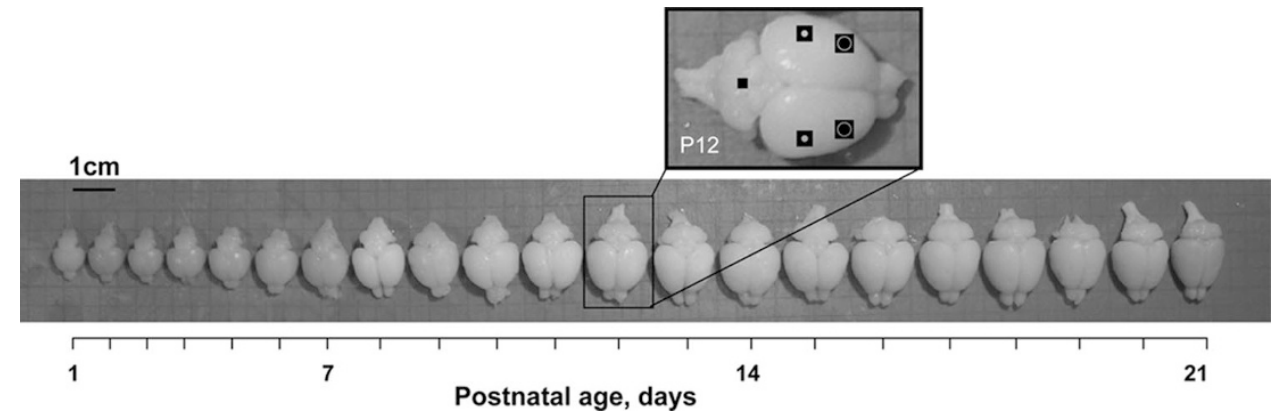

Figure 1. Rat brains at increasing postnatal ages P1-P21. Rate of brain growth peaked around $\mathrm{P} 7$. Brain weight at birth $\approx 0.25 \mathrm{~g}$, by $\mathrm{P} 7 \approx 0.8 \mathrm{~g}, \mathrm{P} 14 \approx 1.5 \mathrm{~g}$, and $\mathrm{P} 21 \approx 1.7 \mathrm{~g}$. The body weight increases steadily form birth $(5$ g) until P21 (34 g). The P10 brain shows

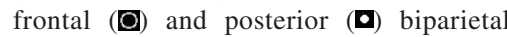
placement of recording and reference electrodes (-) for EEG and aEEG. animals were unsedated and gently swaddled throughout the monitoring period to reduce movement artifact.

Pups were allowed to recover from anesthesia for at least $15 \mathrm{~min}$ before recording. By this time, there was no effect of halothane on the EEG recording. The distance between electrodes varied according to the size of the animal, from $5 \mathrm{~mm}$ on P1, to $12 \mathrm{~mm}$ on P21 (Fig. 1). In pilot recordings (not shown), we found that the bandwidth of the aEEG trace is minimally affected by interelectrode distance in the range $5-12 \mathrm{~mm}$. The biparietal posterior and reference electrode placement was used for machine A (Fig. 1). For machine $\mathrm{B}$, in addition to these three electrodes, a further two anterior electrodes were used (Fig. 1). During recordings, impedance remained between 3 and $9 \mathrm{k} \Omega$. The two monitors differ subtly in terms of filtering and amplification of the raw EEG signal. These new digital machines aim to reproduce the settings of the original analogue machine (Lectromed 5330) by reversed engineering (16) (http://www.brainzinstruments.com/new/BRM2.asp).

Survival. After the last recording, pups were deeply anesthetized and the brains perfusion-fixed, weighed, and photographed. Brain size increased rapidly from P1 $(0.28 \mathrm{~g})$ to P15 (1.81 g) (Fig. 1), with maximal rate of rise around P7 (17). Although body weight continued to increase, brain weight plateaued at P15, as previously published (18).

Trace analysis. Two investigators (E.C. and K.A.), blinded to the age of the animal, independently evaluated the traces. A minimum of $60 \mathrm{~min}$ of noise free recording was analyzed.

Amplitudes of the upper and lower margins were assessed from the compressed aEEG recording $(6 \mathrm{~cm} / \mathrm{h})$. These were determined digitally by plotting a trend line through the median upper peak or lower trough, respectively, on a $200 \%$ enlarged trace on the computer screen. The CFM 6000 was used with standard display for aEEG. The component of the trace lying above $10 \mu \mathrm{V}$ was read with a superimposed semilogarithmic ruler. Bandwidth represents the difference between upper and lower voltage margins.

The longest IBI was evaluated from raw EEG traces run at $30 \mathrm{~mm} / \mathrm{s}$. Periods of movement artifact were excluded from analysis. A burst was defined as electrocortical activity with amplitude $>30 \mu \mathrm{V}$, lasting $>1 \mathrm{~s}$ (19). The five longest IBIs per trace (individual traces lasting at least $1 \mathrm{~h}$ ) were measured and the median calculated.

Statistical analysis. The best-fit correlations (linear or exponential) between variables were analyzed using SPSS 14.0 for Windows (Chicago, Illinois). Where differences were detected between machines, data were split for machine, otherwise, data were combined. The results were not affected by the number of times $(1,2$, or 3$)$ individual animals were used for recording.

\section{RESULTS}

The 110 traces analyzed yielded a median of five traces (range 2-10) for each postnatal day and the machine used (see Methods section) were randomly selected for each recording, machine A: Olympic or B: Brainz. Figure 2 shows typical aEEG and EEG traces at different GAs.

Table 1 shows the data from the linear or exponential regression models used when different variables (the upper and lower voltage margin of the aEEG trace and IBI of the EEG trace) are related using the two different machines.

Upper and lower margin. There is an exponential relationship between the voltage of the lower aEEG margin and postnatal age (with no significant difference between the two machines) (Table 1.2).

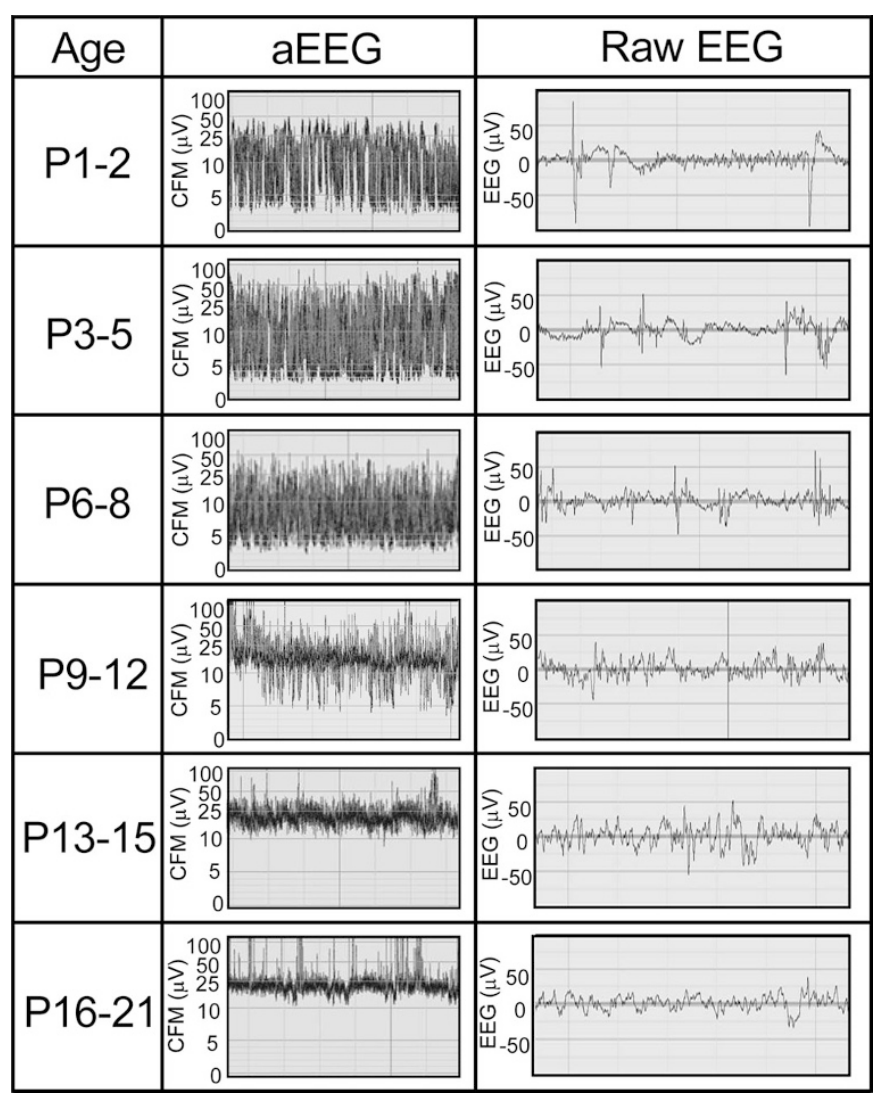

Figure 2. Representative aEEG (trace speed $6 \mathrm{~cm} / \mathrm{h}$ ) and raw EEG traces (30 $\mathrm{mm} / \mathrm{s}$ ) from $\mathrm{P} 1$ to $\mathrm{P} 21$. With increasing age, trace activity becomes more continuous; this can be seen in both aEEG and raw EEG traces. The aEEG lower voltage margin rises faster than the aEEG upper voltage margin and the overall trace bandwidth narrows with increasing postnatal age. Note that for $\mathrm{P} 9-\mathrm{P} 12$ there are frequent low amplitude bursts, as one would expect from the corresponding IBI.

Tables 1.3 and 1.4 shows the relationship between the upper margin and postnatal age using a linear (Table 1.3, machine B read $3.37 \mu \mathrm{V}$ lower than machine A) or an exponential (Table 1.4) model, respectively. The exponential model offers a marginally better fit. The individual data points for the lower and upper margins versus postnatal age are displayed in Figure 3.

Figure 4 shows a graphic representation of how the bandwidth of the aEEG trace develops from birth to 3 wk of age in the aEEG semilogarithmic plot.

Interburst interval. The median of the longest five IBIs per trace decreased linearly from P1 to P21 (Fig. 5). Data from the 
Table 1. Analysis of lower and upper margin of the semilogarithmic voltage scale individually and related to either postnatal age or the median longest IBI per trace

\begin{tabular}{ccccccc}
\hline$b_{0}$ & $\mathrm{SD}\left(b_{0}\right)$ & $b_{1}$ & $\mathrm{SD}\left(b_{1}\right)$ & $b_{2}$ & $\mathrm{SD}\left(b_{2}\right)$ & Machine \\
\hline 1.1. IBI (s) & as a linear function of age & & \\
114.1 & 7.48 & -5.24 & 0.365 & 21.13 & 4.191 & $\mathrm{~A}+\mathrm{B}$ \\
132.2 & 6.06 & -4.96 & 0.489 & & $\mathrm{~A}$ \\
159.4 & 7.39 & -5.48 & 0.541 & $\mathrm{~B}$ \\
1.2 Amplitude $(\mu \mathrm{V})$ & of lower margin as an exponential function of age \\
2.899 & 0.168 & 0.093 & 0.004 & $\mathrm{~A}+\mathrm{B}$ \\
2.891 & 0.258 & 0.10 & 0.007 & $\mathrm{~A}$ \\
2.79 & 0.185 & 0.090 & 0.005 & $\mathrm{~B}$ \\
1.3 Amplitude $(\mu \mathrm{V})$ & of upper margin as a linear function of age \\
17.88 & 2.58 & 0.95 & 0.126 & -3.37 & 1.446 & $\mathrm{~A}+\mathrm{B}$ \\
16.01 & 2.53 & 0.82 & 0.204 & $\mathrm{~A}$ \\
9.68 & 2.04 & 1.07 & 0.149 & $\mathrm{~B}$ \\
1.4 Amplitude $(\mu \mathrm{V})$ & of upper margin as an exponential function of age \\
14.232 & 0.896 & 0.039 & 0.005 & $\mathrm{~A}+\mathrm{B}$ \\
16.112 & 1.569 & 0.034 & 0.008 & $\mathrm{~A}$ \\
12.174 & 0.893 & 0.046 & 0.005 & $\mathrm{~B}$ \\
1.5 IBI (s) as an exponential function of the lower aEEG $(\mu \mathrm{V})$ & trace \\
153.77 & 8.716 & -0.070 & 0.005 & $\mathrm{~A}+\mathrm{B}$ \\
133.82 & 10.218 & -0.062 & 0.006 & $\mathrm{~A}$ \\
180.25 & 14.600 & -0.080 & 0.007 & $\mathrm{~B}$ \\
\hline
\end{tabular}

Results from linear or exponential regression are shown using the following models (SPSS 14.0).

Linear: $\mathrm{Y}=b_{0}+b_{1} * X_{1}+b_{2} * X_{2} \quad$ Exponential: $\mathrm{Y}=b_{0} * \exp \left(b_{1} * X\right)$

Machine $\mathrm{A}=1$, machine $\mathrm{B}=2$

When $X_{2}=1$, machine $=\mathrm{A}$

When $X_{2}=2$, machine $=\mathrm{B}$

in 1.1-1.4, $X_{1}$ is days of life

in $1.5, X_{1}$ is the aEEG lower amplitude voltage.

Example: using values from 1.3, A + B

$$
\mathrm{Y}=17.88+0.95 * X_{1}+-3.37 * X_{2}
$$

If $X_{1}=0, \quad \mathrm{Y}=17.88-3.37 * 1=14.51$ for machine A

and $\quad \mathrm{Y}=17.88-3.37 * 2=10.34$ for machine $\mathrm{B}$.

Here, we have forced the relationship for A and B to have the same slope. $b_{2} \mathrm{SD}=1.446$. Two $\mathrm{SD}=2.89$ which is $<3.37$. Thus, we conclude that $\mathrm{A}$ and $\mathrm{B}$ are significantly different.

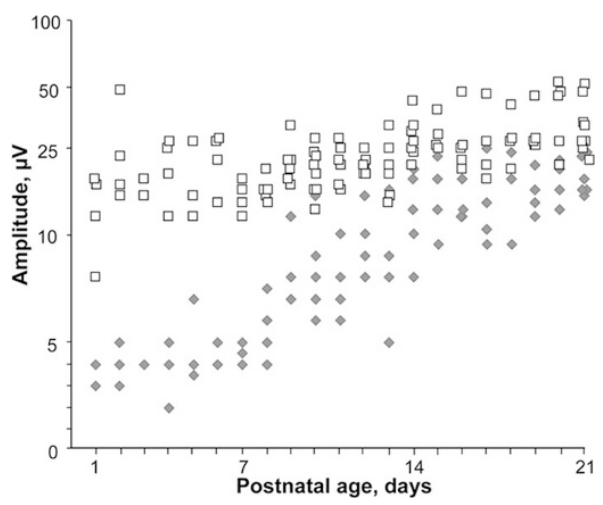

Figure 3. Scatter plot of minimum $(\diamond)$ and maximum $(\square)$ aEEG voltage amplitude for all animals from P1 to P21. The $y$ axis is semilogarithmic, as in standard aEEG recordings. See Tables $1.2-1.4$ for equations of best fit.

linear regression between the longest IBI and postnatal age are given in Table 1.1. IBI values are $21 \mathrm{~s}$ (95\% CI 13-29) longer in machine $\mathrm{B}$ than in machine $\mathrm{A}, p<0.001$. Table 1.5 shows that the aEEG lower margin increased exponentially as IBI decreased and with no difference between the two machines. The raw data are displayed in Figure 6.

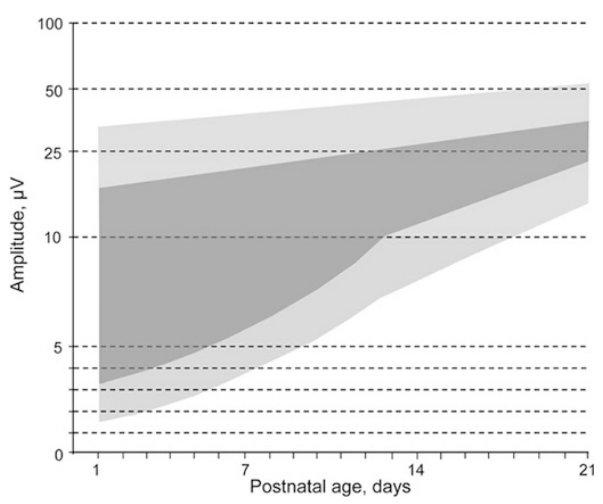

Figure 4. Graphic representation of the development of aEEG upper and lower voltage margin (dark gray, linear fit). Light gray represents two SD. The $\mathrm{y}$-axis is linear $0-10 \mu \mathrm{V}$, then logarithmic.

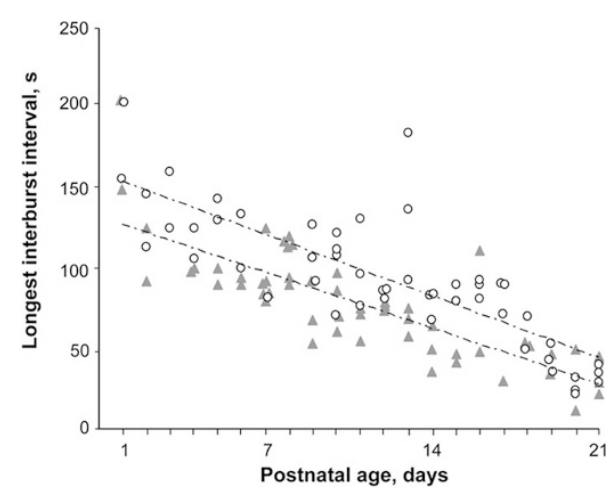

Figure 5. Linear relationship between the median longest IBIs and age, split for machine (machine A- $\Delta$, machine B-O. Values are significantly higher for machine B (formulae in Table 1.1).

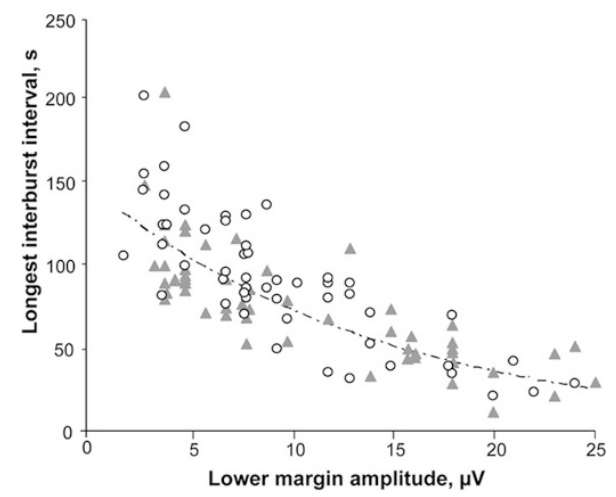

Figure 6. Exponential relationship between the median longest IBIs and the aEEG lower margin (machine A- $\triangle$, machine B-O. Values do not differ between machines (formulae in Table 1.5).

\section{DISCUSSION}

The objective of this study was to describe the development of aEEG and maximum IBI in rats from P1 to P21. The rat pup is used at different ages to mimic human brain injury models across a range of developmental ages. aEEG is increasingly used in humans to assess maturation and injury. Hence, we sought to suggest comparative ages for the rat and the human newborn based on aEEG pattern and the longest IBI. Although detailed descriptions of rat EEG maturation from P9 to P30 are available (11), serial aEEG changes and changes in the longest IBI have not previously been described. It is assumed 
that the P7 rat, widely used in models of human neonatal brain injury, is equivalent in maturation to the near-term human newborn (20).

aEEG background activity in the term infant was first classified by Lena Westas as "continuous" (without low voltage periods), "somewhat continuous," "discontinuous" [with low voltage periods $(<5 \mu \mathrm{V})]$, or "flat" $(21,22)$. A number of studies have evaluated aEEG in term and preterm neonates (22-27).

In a study examining aEEG in preterm infants, 119 aEEG recordings were obtained from 31 infants with postmenstrual age 25-32 wk. Discontinuous tracings were only observed before $33 \mathrm{wk}$. In a few individuals continuous tracings appeared at a postmenstrual age of $27 \mathrm{wk}$ (25). In another study evaluating 146 traces from 30 infants between 24 and 39 wk GA, excluding infants with severe intraventricular hemorrhage or encephalopathy, traces were discontinuous from 24 to 26 wk. Continuity increased with age, and all traces beyond 30-31 wk were continuous (22). Olischar et al. (24) confirmed the correlation between GA and duration of continuous aEEG activity in "normal" preterm neonates under 30-wk GA previously shown using EEG (28). These studies were performed using subjective assessment of compressed aEEG paper traces $(6 \mathrm{~cm} / \mathrm{h})$ where the resolution for discontinuity is limited by the tracing pen's width. The trace appears "dense" when there are more than 20 spikes/10 min at a recording speed of $1 \mathrm{~mm} / \mathrm{min}$. It is therefore difficult to count IBIs shorter than $30 \mathrm{~s}$ without digital equipment. Thus, continuity was likely to be overestimated in old paper traces compared with digital analyses.

We tried to apply a published clinical continuity score for aEEG maturation described by Sisman and Burdjalov $(22,25)$. In their three-tier scoring system, the traces were subjectively classified into continuous, intermediate, or discontinuous based on the overall trace density (old paper trace). Cyclic changes in bandwidth (SWC) were given a five-point score $(22,25)$. We found that a subjective scoring system based on the compressed traces lacks sensitivity in an experimental setting, as compared to the new EEG/aEEG equipment available.

Figures 3, 4, and 5 indicate significant discontinuity until P8. In our study, the lower and upper margin of the aEEG voltage trace in the $\mathrm{P} 1$ rat is broadly equivalent to the pattern seen at 23-wk gestation in the human, P6 to 27 wk $(28,29)$, P7 to 30-32 wk, and P10 to 40-42-wk GA.

Duration of IBIs, often defined as the interval between spikes of activity $>30 \mu \mathrm{V}(19,29,30)$, is a surrogate measure of electroencephalographic continuity. As both aEEG machines that were used also display raw EEG, we were able to determine the duration of the longest IBIs $>30 \mu \mathrm{V}$ in each trace by analyzing the trace at $30 \mathrm{~mm} / \mathrm{s}$. This allowed us to compare our results with a large body of literature describing variation in IBI with postnatal age.

Several authors have shown that maximal IBI decreases with increasing GA (21,31-34). The actual values for maximal IBI at specified time points vary in these studies. The variations are partly related to exclusion criteria, as not all studies $(29,35)$ applied rigorous selection and long-term follow-up to ensure that only those premature infants progressing to normal neurologic outcome are reported. Slight differences in defining the size and duration of bursts are also evident. One study (28) recorded EEGs for $24 \mathrm{~h}$ and then only measured IBIs on epochs from the most discontinuous segments. In addition, the greater physiologic stability allowed by progressive improvements in neonatal care seems to have lowered corresponding IBI values. Dreyfus-Brisac reported an IBI duration of $3 \mathrm{~min}$ at 24-28 wk GA in 1962 (31); this decreased to $88 \mathrm{~s}$ for $27-29 \mathrm{wk}$ (34) and subsequently to $60 \mathrm{~s}$ at 27 wk (28). A rapid change in IBI from 26 to 29 wk GA, with a slower decrease from 29 to $38 \mathrm{wk}$ has been described for the human preterm neonate (33).

In our rat study, the mean linear fit of the longest IBIs decreased by $5.2 \mathrm{~s} / \mathrm{d}$, from $145 \mathrm{~s}$ on average at P1, to $34 \mathrm{~s}$ at $\mathrm{P} 21$. We read systematically higher IBI values from machine $\mathrm{B}$, which may be due to differences in the filter settings. The absolute values are less important than the finding of a linear decline in IBI with age. The absolute IBI values are higher than those presented from humans. This is likely because of our high burst cut-off value of $30 \mu \mathrm{V}$. We used the same burst definition used for human babies to get data comparable with human studies. Bursts in rats are often of lower voltage than in human newborns and this could account for longer IBIs.

IBIs are virtually absent in the EEGs of normal full-term infants (32). Two studies have evaluated the significance of excessive EEG discontinuity in the term infant $(36,37)$. In these studies, bursts were defined as minimum amplitudes of 50 or $45 \mu \mathrm{V}$, respectively. In the first study, $89 \%$ of term neonates with maximal IBI duration above $40 \mathrm{~s}$ had an unfavorable outcome (37). Biagioni et al. showed that it is the maximal IBI that relates most closely to both outcome and severity of neonatal encephalopathy. The mean value was lower than $10 \mathrm{~s}$ in normal infants. All deceased infants demonstrated maximal values over $40 \mathrm{~s}$. All neonates with a discontinuous EEG after $11 \mathrm{~d}$ of age developed severe neurologic sequelae or died (36).

In contrast, in our study, rats demonstrated a degree of discontinuity, with evidence of quiet interburst periods, through the period of term equivalence (which we suggest is P10-P12) and up to P21. Cortical development, specifically the degree of cortical folding, correlates well with IBI in the human neonate (38). As the rat brain is nongyrencephalic, fundamental differences between background activity in the mature rat and human EEGs are not surprising.

Values for the aEEG upper margin show a good correlation with age in the premature neonate, reaching a maximum at 36-37 wk GA (22). In our study, values for both upper and lower aEEG margins correlated well with GA (Fig. 5) fitting an exponential equation (Table 1). The two machines read similar levels for both the lower and upper borders, with machine $\mathrm{B}$ obtaining $3.37 \mu \mathrm{V}$ lower values for the upper margin of aEEG. In comparison to the original Lectromed paper trace, slightly lower borders have also been reported using machine B in humans (39).

The lower margin of aEEG is normally above $5 \mu \mathrm{V}$ in infants older than 30-wk GA. This observation may suggest that $\mathrm{P} 7$ in the rat may be equivalent to $30-32 \mathrm{wk}$ in the human. The lower border of aEEG rose above $5 \mu \mathrm{V}$ after P7 suggest- 
ing that the term equivalent aEEG of the rat is P10-P12. The correlation of bandwidth with postnatal age, evident in human neonatal aEEG studies $(22,40)$, was also evident in this study (Figs. 4 and 5). aEEG in humans has shown cyclic changes in bandwidth, corresponding to periods of quiet or active sleep. In humans, SWC is a sign of maturation and wellbeing and is evident by $30-31 \mathrm{wk}$ GA (40). We were unable to identify any aEEG changes related to SWC in this study, possibly because rats are known to have very brief sleep wake cycles (26), lasting only a few minutes.

Dobbing and Sands (17) identified the point of maximal brain growth to occur at term in humans and at $\mathrm{P} 7$ in rats. Brain growth peaked in our study around P7 (Fig. 2). Subsequent studies have shown maximal white matter vulnerability in humans at 24-32 wk, when O4+O1-preoligodendrocytes predominate. In the rat, this population predominates at $\mathrm{P} 4$, with a subsequent shift to $\mathrm{O} 4+\mathrm{O} 1+$ cells by P7 (12). Romijn et al. studied four developmental parameters (development of glutamate decarboxylase, choline acetyltransferase electrical activity, and numerical synapse formation), concluding that the peak change of these parameters is at term for the human neonate and ranges from P7.4 to P20.4 in the rat. They argued that the best overall correlation between the rat and human maturation was given by EEG data, suggesting that term equivalence occurs at P12-P13 (41).

aEEG is a clinically relevant tool that is therefore useful in the evaluation of brain maturational changes in translational research. Our data suggests that the P7 rat is equivalent to 30-32-wk gestation and P10 closer to 40-42-wk gestation in the human.

\section{REFERENCES}

1. Hellström-Westas L, Rosén I 2006 Continuous brain-function monitoring: State of the art in clinical practice. Semin Fetal Neonatal Med 11:503-511

2. McBride MC, Laroia N, Guillet R 2000 Electrographic seizures in neonates correlate with poor neurodevelopmental outcome. Neurology 55:506-513

3. Sinclair DB, Campbell M, Byrne P, Prasertsom W, Robertson CM 1999 EEG and long-term outcome of term infants with neonatal hypoxic-ischemic encephalopathy. Clin Neurophysiol 110:655-659

4. Bona E, Hagberg H, Loberg EM, Bagenholm R, Thoresen M 1998 Protective effects of moderate hypothermia after neonatal hypoxia-ischemia: short- and long-term outcome. Pediatr Res 43:738-745

5. Cherian SS, Love S, Silver IA, Porter HJ, Whitelaw AG, Thoresen M 2003 Posthemorrhagic ventricular dilation in the neonate: development and characterization of a rat model. J Neuropathol Exp Neurol 62:292-303

6. Jensen FE, Applegate CD, Holtzman D, Belin TR, Burchfiel JL 1991 Epileptogenic effect of hypoxia in the immature rodent brain. Ann Neurol 29:629-637

7. Pulsinelli WA, Brierley JB 1979 A new model of bilateral hemispheric ischemia in the unanesthetized rat. Stroke 10:267-272

8. Rice JE III, Vannucci RC, Brierley JB 1981 The influence of immaturity on hypoxic-ischemic brain damage in the rat. Ann Neurol 9:131-141

9. Blumberg RM, Cady EB, Wigglesworth JS, McKenzie JE, Edwards AD 1997 Relation between delayed impairment of cerebral energy metabolism and infarction following transient focal hypoxia-ischaemia in the developing brain. Exp Brain Res 113:130-137

10. Mu D, Jiang X, Sheldon RA, Fox CK, Hamrick SE, Vexler ZS, Ferriero DM 2003 Regulation of hypoxia-inducible factor 1alpha and induction of vascular endothelial growth factor in a rat neonatal stroke model. Neurobiol Dis 14:524-534

11. Gramsbergen A 1976 The development of the EEG in the rat. Dev Psychobiol 9:501-515

12. Hagberg H, Peebles D, Mallard C 2002 Models of white matter injury: comparison of infectious, hypoxic-ischemic, and excitotoxic insults. Ment Retard Dev Disabil Res Rev 8:30-38
13. Skoff RP, Ghandour MS, Knapp PE 1994 Postmitotic oligodendrocytes generated during postnatal cerebral development are derived from proliferation of immature oligodendrocytes. Glia 12:12-23

14. Conradi NG, Muntzing K, Sourander P, Hamberger A 1984 Effect of ambient temperature on rectal temperature in normal and malnourished rats during early postnatal development. Acta Physiol Scand 121:147-153

15. Dingley J, Hobbs C, Ferguson J, Stone J, Thoresen M 2008 Xenon/hypothermia neuroprotection regimes in spontaneously breathing neonatal rats after hypoxicischemic insult: the respiratory and sedative effects. Anesth Analg 106:916-923; table of contents

16. Maynard D, Prior PF, Scott DF 1969 Device for continuous monitoring of cerebral activity in resuscitated patients. BMJ 4:545-546

17. Dobbing J, Sands J 1979 Comparative aspects of the brain growth spurt. Early Hum Dev 3:79-83

18. Dobbing J, Sands J 1973 Quantitative growth and development of human brain. Arch Dis Child 48:757-767

19. Selton D, Andre M, Hascoet JM 2000 Normal EEG in very premature infants: reference criteria. Clin Neurophysiol 111:2116-2124

20. Hagberg H, Bona E, Gilland E, Puka-Sundvall M 1997 Hypoxia-ischaemia model in the 7-day-old rat: possibilities and shortcomings. Acta Paediatr Suppl 422:85-88

21. Hellstrom-Westas L, Klette H, Thorngren-Jerneck K, Rosen I 2001 Early prediction of outcome with aEEG in preterm infants with large intraventricular hemorrhages. Neuropediatrics 32:319-324

22. Burdjalov VF, Baumgart S, Spitzer AR 2003 Cerebral function monitoring: a new scoring system for the evaluation of brain maturation in neonates. Pediatrics 112:855-861

23. Hellstrom-Westas L, Rosen I, Svenningsen NW 1991 Cerebral function monitoring during the first week of life in extremely small low birthweight (ESLBW) infants. Neuropediatrics 22:27-32

24. Olischar M, Klebermass K, Kuhle S, Hulek M, Kohlhauser C, Rucklinger E, Pollak A, Weninger M 2004 Reference values for amplitude-integrated electroencephalographic activity in preterm infants younger than 30 weeks' gestational age. Pediatrics 113:e61-e66

25. Sisman J, Campbell DE, Brion LP 2005 Amplitude-integrated EEG in preterm infants: maturation of background pattern and amplitude voltage with postmenstrual age and gestational age. J Perinatol 25:391-396

26. Spitzmiller RE, Phillips T, Meinzen-Derr J, Hoath SB 2007 Amplitude-integrated EEG is useful in predicting neurodevelopmental outcome in full-term infants with hypoxic-ischemic encephalopathy: a meta-analysis. J Child Neurol 22:1069-1078

27. Viniker DA, Maynard DE, Scott DF 1984 Cerebral function monitor studies in neonates. Clin Electroencephalogr 15:185-192

28. Connell JA, Oozeer R, Dubowitz V 1987 Continuous 4-channel EEG monitoring: a guide to interpretation, with normal values, in preterm infants. Neuropediatrics 18:138-145

29. Hayakawa M, Okumura A, Hayakawa F, Watanabe K, Ohshiro M, Kato Y, Takahashi R, Tauchi N 2001 Background electroencephalographic (EEG) activities of very preterm infants born at less than 27 weeks gestation: a study on the degree of continuity. Arch Dis Child Fetal Neonatal Ed 84:F163-F167

30. Biagioni E, Bartalena L, Boldrini A, Cioni G, Giancola S, Ipata AE 1994 Background EEG activity in preterm infants: correlation of outcome with selected maturational features. Electroencephalogr Clin Neurophysiol 91:154-162

31. Dreyfus-Brisac C 1962 The electroencephalogram of the premature infant. World Neurol 3:5-15

32. Hahn JS, Monyer H, Tharp BR 1989 Interburst interval measurements in the EEGs of premature infants with normal neurological outcome. Electroencephalogr Clin Neurophysiol 73:410-418

33. Hughes JR, Fino J, Gagnon L 1983 Periods of activity and quiescence in the premature EEG. Neuropediatrics 14:66-72

34. Monod N, Dreyfus-Brisac C, Ducas P, Mayer M 1960 [The EEG of the newborn a term. Comparative study in the newborn infant in cephalic presentation and breech presentation]. Rev Prat 102:375-379

35. Anderson CM, Torres F, Faoro A 1985 The EEG of the early premature. Electroencephalogr Clin Neurophysiol 60:95-105

36. Biagioni E, Bartalena L, Boldrini A, Pieri R, Cioni G 1999 Constantly discontinuous EEG patterns in full-term neonates with hypoxic-ischaemic encephalopathy. Clin Neurophysiol 110:1510-1515

37. Menache CC, Bourgeois BF, Volpe JJ 2002 Prognostic value of neonatal discontinuous EEG. Pediatr Neurol 27:93-101

38. Biagioni E, Frisone MF, Laroche S, Kapetanakis BA, Ricci D, Adeyi-Obe M, Lewis H, Kennea N, Cioni G, Cowan F, Rutherford M, Azzopardi D, Mercuri E 2007 Maturation of cerebral electrical activity and development of cortical folding in young very preterm infants. Clin Neurophysiol 118:53-59

39. Shah DK, Lavery S, Doyle LW, Wong C, McDougall P, Inder TE 2006 Use of 2-channel bedside electroencephalogram monitoring in term-born encephalopathic infants related to cerebral injury defined by magnetic resonance imaging. Pediatrics 118:47-55

40. Thornberg E, Thiringer K 1990 Normal pattern of the cerebral function monitor trace in term and preterm neonates. Acta Paediatr Scand 79:20-25

41. Romijn HJ, Hofman MA, Gramsbergen A 1991 At what age is the developing cerebral cortex of the rat comparable to that of the full-term newborn human baby? Early Hum Dev 26:61-67 